\title{
Temperamento y carácter en adictos a cocaína y a opiáceos
}

\author{
Estibaliz Barron Pardo*, Manuel Mateos Agut**, Itziar Martínez Villate*** \\ * Directora. Fundación Gizakia. Bilbao. \\ * Coordinador. Hospital de Día del Servicio de Psiquiatría del Hospital Divino Valles. Burgos. \\ *** Responsable Área de Drogodependencias. Fundación Gizakia. Bilbao.

\section{RESUMEN}

Se estudian, a partir de la aplicación del Inventario de Temperamento y Carácter (TCI) de Cloninger, las dimensiones de personalidad de una muestra de pacientes dependientes a sustancias (DSM-IV) en tratamiento de rehabilitación. Se comparan los resultados con los obtenidos por un grupo control extraído de la población general. Los resultados indican que existen diferencias significativas entre los rasgos de personalidad que presentan, tanto el subgrupo de dependientes a opiáceos como el de dependientes a cocaína, una vez comparados con la población general.

Comparado con el grupo de control, el grupo de heroinómanos obtiene, puntuaciones más bajas en las escalas de Dependencia de la Recompensa (RD), Autodirección (S) y Cooperación (C). Así como divergencias significativas en tres de las cuatro subescalas de Evitación del Riesgo $(\uparrow \mathrm{HA} 1$; $\downarrow H A 2 ; \uparrow H A 3)$ y en una de las correspondientes a Búsqueda de Novedades ( $\uparrow$ NS3).

El grupo de cocainómanos presenta mayor Búsqueda de Novedades (NS), menor Dependencia de la Recompensa, menor Autodirección (S) y menor Cooperación (C). También presentan significación los resultados de una de las subescalas de Evitación del Riesgo ( $\downarrow$ HA2).

Comparados entre sí el grupo de cocaínomanos y el de heroinómanos se encuentra que difieren significativamente en dos de las subescalas del Temperamento: una perteneciente a la escala de Búsqueda de Novedades (NS4) y otra a la de Evitación de Riesgo (HA3).

Palabras clave: Personalidad, Dependencia a opiáceos, Dependencia a cocaína, TCl, Escala de Cloninger, DSM-IV .

\section{ABSTRACT}

The study was established to investigate the personality dimensions of a sample of substance dependents (DSM-IV) undergoing rehabilitation treatment using the Cloninger's Temperament and Character Inventory (TCl). Comparison of the results with those of a control group extracted from the general population confirmed that there were significant differences between the personality features in both the opiate and cocaine subgroups, compared with those from the general population.

Comparing the control group with the heroin dependents, the results showed lower scores on the scales for reward dependence (RD), self-directedness (SD) and Cooperation (C), than the control group. Significant divergences were also found on three of the four harm avoidance scales ( $\uparrow$ HA1; $\downarrow H A 2 ; \uparrow H A 3)$ and on one of the novelty seeking scales ( $\uparrow$ NS3).

In the case of the group of cocaine dependents, there was a higher level of novelty seeking but lower levels of reward dependency (RD), self-directedness (SD) and cooperation. The results of one of the harm avoidance subscales $(\downarrow \mathrm{HA} 2)$ were also significant. Finally, comparing the cocaine dependents and the heroin dependents, it was found that they differed significantly on two of the temperament subscales, one forming part of novelty seeking (NS4) and the other part of harm avoidance (HA3).

Key Words: Personality, Opiate dependents. Cocaine dependents, Cloninger, TCl, DSM-IV.

\section{INTRODUCCIÓN}

I panorama actual de las drogodependencias se caracteriza por la diversidad tanto de los perfiles de las personas drogodependientes como de los posibles tratamientos a instaurar en cada caso. Superadas ya las posturas de defensa de modelos únicos de intervención, la correcta asignación de los pacientes al tratamiento debe contemplar no solo las categorías diagnósticas definidas en los manuales al uso, sino también orientaciones clínicas que permitan ajustar más las estrategias de intervención.

El estudio que a continuación se presenta, se desarrolla precisamente a partir de la incorporación de una 
prueba, el Inventario de Personalidad del Temperamento y el Carácter de Cloninger ( $\mathrm{TCl}$ ), en el protocolo de diagnóstico y definición de itinerario terapéutico, de los pacientes que acuden a tratamiento al programa de rehabilitación de drogodependientes Proyecto Hombre de Bizkaia. El estudio se desarrolla gracias a la colaboración entre la entidad promotora de dicho programa, Fundación Gizakia y el Hospital Divino Valles de Burgos.

Los distintos enfoques en el abordaje de las drogodependencias se fundamentan en modelos teóricos diferentes que marcan la orientación de los tratamientos. Sin embargo, sea cual sea el modelo de referencia, existe un consenso subyacente en cuanto a la aceptación de la multicausalidad en la génesis de la drogodependencia, y en la estrecha relación de las variables biológicas, psicológicas y sociales intervinientes en cada caso concreto.

Si observamos las diferentes líneas de investigación abiertas en torno al tratamiento de las drogodependencias: el desarrollo de nuevos psicofármacos; la búsqueda de causas genéticas de la adicción; el reconocimiento de la necesidad de un abordaje psicoterapéutico que provoque cambios estructurales; la definición de programas de prevención de recaídas; vemos como cada una de ellas aborda alguno de los factores del enfoque biopsicosocial.

En este sentido el modelo psicobiológico de la personalidad de Cloninger nos parece de gran interés para el ámbito de las drogodependencias dado que integra tanto los aspectos constitucionales de la personalidad como los adquiridos. Además, la prueba que desarrollan a partir de este modelo para evaluar la personalidad (TCl), distingue entre rasgos heredados y rasgos añadidos durante el desarrollo a partir de las propias experiencias, con lo que incorpora orientaciones muy interesantes, desde el punto de vista clínico, sobre las posibilidades de cambio a través de enfoques más psicoterapéuticos o biologicistas (Mateos y De la Gándara, 2002)

Aunque durante años la búsqueda de un perfil de personalidad característico de la población drogodependiente marcó la línea de muchos estudios, hoy día, dentro del campo de la toxicomanía, se admite ampliamente que no existe una personalidad adictiva, ni un único tipo de personalidad que predispone a la adicción (Franques et al. 2000).

Numerosos estudios describen que en el colectivo de toxicómanos se da una prevalencia de trastornos de personalidad muy superior a la media de la población general; sobre todo en lo que hace referencia a los trastornos de personalidad antisocial (Craig 2000) y (Razzouk 2000). Sin embargo, hay autores que apuntan la posibilidad de que esta tasa esté sobredimensionada (Bolinches et al. 2002).
Quizás haya que considerar que el tipo de vida desarrollada después de la génesis de la drogodependencia, en muchas ocasiones al margen de la ley, mediatiza el diagnóstico de personalidad elevando a categorías, lo que bien pudieran ser rasgos, pero en todo caso, todo ello indica la necesidad de precisar mejor nuestro diagnóstico de la personalidad.

\section{MATERIAL Y MÉTODO}

\section{MUESTRA}

Se estudia una muestra de 117 varones adscritos a tratamiento durante el año 2002, en el programa de rehabilitación de drogodependientes Proyecto Hombre de Bizkaia, promovido por la Fundación Gizakia.

Todos ellos reúnen los criterios (DSM-IV) para el diagnóstico de dependencia a sustancias. El 41.9\% (49) de los casos fueron diagnosticados de dependencia a opiáceos, siendo la heroína la droga principal de consumo. El 58.1\% restante (68) presentaba dependencia a cocaína. En el 73.5 \% de los casos hay, además, consumo de otras sustancias.

El 93,2 \% proceden de núcleos urbanos de la provincia de Bizkaia. La edad media de los heroinómanos 34.9 (d. tip. 5.9), es algo superior a la del grupo de cocainómanos 31.7 (d. tip. 6.3).

En cuanto al estado civil, más de la mitad de la muestra está soltera (66.7 \%), el 19.6 \% están casados o conviven con su pareja de manera estable y el $13.7 \%$ son separados o divorciados.

En relación con el nivel de estudios, el 23.9 \% abandonaron los estudios en el nivel de estudios primarios, el $56.4 \%$ obtuvo el Graduado Escolar, el $6 \%$ ha completado FPI, el $2.6 \%$ FPII, el 6.8 \% alcanzó el nivel de COU y el $4.3 \%$ tiene estudios superiores. La proporción de personas que han superado el nivel de graduado escolar se encuentra en porcentajes mayores en el grupo de cocainómanos.

La razón de que se haya excluido de este estudio a la población drogodependiente femenina responde a razones de significación estadística, dado que durante el tiempo en que se recoge la muestra menos del $10 \%$ del colectivo atendido son mujeres. Los autores han optado por homogeneizar la muestra limitando el estudio a la población masculina, para evitar posibles sesgos por razones de género en los resultados de las medidas realizadas por el $\mathrm{TCl}$.

\section{MATERIAL}


Se evaluó la personalidad de la muestra y del grupo control mediante el Inventario de Temperamento y Carácter de Cloninger (TCI). Este instrumento está elaborado basándose en el modelo de la personalidad desarrollado por el mismo autor y permite analizar de manera cuantitativa la personalidad.

Se utilizó la versión traducida al castellano por Carmen Bayón y modificada por Sanz et al. Aunque el TCl se encuentra aún en fase de validación para población española, recientes estudios psicométricos (Gutiérrez et al 2001) concluyen que la versión española presenta una buena validez y fiabilidad. Manejamos la baremación obtenida en población general y población psiquiátrica publicada en el estudio de Mateos y De la Gándara (2001).

El modelo psicobiológico de la personalidad de Cloninger está construido a partir de conceptos neurobiológicos y psicopatológicos y permite evaluar tanto la personalidad normal como la patológica. Es un modelo que intenta integrar el substrato biológico de la personalidad con los elementos añadidos durante el desarrollo y por la experiencia sociocultural. Rescata los conceptos de Temperamento (definido como la predisposición con la que nacemos y que se refiere a los aspectos de la personalidad que están ligados a sistemas neurológicos definidos, se manifiestan precozmente en la vida, se mantienen estables a lo largo de la misma y consti- tuyen inclinaciones probablemente heredables que son modificables sobre todo biológicamente) y Carácter (que hace referencia a los autoconceptos que influyen en nuestras intenciones y actitudes, se desarrolla por medio del aprendizaje introspectivo, está influido por el ambiente sociocultural y familiar y es modificable por medio de la psicoterapia). Así, describe siete dimensiones de la personalidad: cuatro referidas al Temperamento y tres al Carácter (Mateos, De la Gándara, 2001).

$\mathrm{El} \mathrm{TCl}$ es un cuestionario autoaplicado que consta de 240 ítems, a los que se responde verdadero o falso. Las puntuaciones se dividen en cuatro escalas que evalúan el Temperamento y tres que evalúan el Carácter. Cada una de estas escalas está dividida a su vez en varias subescalas. (Tablas 1 y 2 ).

\section{PROCEDIMIENTO}

Se dividió la muestra en dos subgrupos formados por las personas diagnosticadas de dependencia a heroína y de dependencia a cocaína respectivamente. Se establecieron grupos control para ambos subgrupos obtenidos en población general no psiquiátrica, formada por personal no facultativo de un hospital general y su familiares y un grupo de estudiantes de una escuela

\section{Tabla $n^{\circ} 1$. Escalas y Subescalas del Temperamento}

NS Búsqueda de novedades: Inclinación heredable hacia una intensa excitación como respuesta a estímulos que indican potenciales recompensas o alivio del dolor. Función conductual de la actividad Dopaminérgica mesolímbica.

NS1 Rigidez estoica vs Excitabilidad exploratoria

NS2 Reflexión vs Impulsibidad

NS3 Reserva vs Extravagancia

NS4 Reglamentación estricta vs Desorden

HA Evitación del riesgo: Inclinación heredable a responder intensamente a las señales de estímulos aversivos, inhibiendo la respuesta para evitar el castigo. Función conductual de la actividad Serotoninérgica.

HA1 Optimismo vs Ansiedad anticipatoria

HA2 Confianza vs Miedo a la Incertidumbre

HA3 Gregarismo vs Timidez

HA4 Vigor vs Fatigabilidad

RD Dependencia de la recompensa: Inclinación heredable a responder intensamente a los signos de recompensa y a mantener conductas asociadas con premios o evitación de castigos. Regulada por la Noradrenalina.

RD1 Insensibilidad vs Sentimentalidad

RD3 Desapego vs Apego

RD4 Independencia vs Dependencia

P Persistencia: Mide la perseverancia en la conducta a pesar de la frustración y la fatiga. Probablemente relacionada con circuitos noradrenérgicos

RD2 Falta de persistencia vs persistencia 


\section{Tabla $\mathbf{n}^{\circ}$ 2. Escalas y Subescalas del Carácter}

S Autodirección: Mide la habilidad de una persona para controlar, regular y adaptar su conducta de acuerdo a valores y metas escogidos individualmente.

S1 Proyección de la culpa vs Responsabilidad

S2 Ausencia de metas vs Determinación

S3 Inercia vs Con Recursos

S4 Competición vs autoaceptación

S5 Malos hábitos vs Hábitos congruentes

C Cooperación: Mide las diferencias individuales en la capacidad de aceptar e identificarse con otras personas.

C1 Intolerancia social vs Aceptación social

C2 Insensibilidad vs Empatía

C3 Egoísmo vs Tendencia a ayudar

C4 Venganza vs Compasión

C5 Oportunismo vs Con principios

ST Autotranscendencia: Identificación del individuo con un todo, del cual procede y del cual es parte esencial.

ST1 Cohibición vs Abstracción

ST2 Autodiferenciación vs Identificación transpersonal

ST3 Materialismo racional vs Espiritualidad

de magisterio. Se equilibraron los grupos con relación a las variables de edad y sexo.

Se intentó que los grupos de control y de estudio, se aproximaran en relación con la variable de estudios, aunque destacamos que no fue posible lograr que fueran totalmente homogéneos dado que en la población general, para los mismos rangos de edad, encontramos niveles de estudios algo superiores a los de la muestra estudiada.

La prueba se pasó al grupo de estudio cuando llevaban al menos un mes en tratamiento. Se corrigieron las pruebas mediante la aplicación informática para la corrección del TCl (Mateos y De la Gándara, 2001).

Se compararon las medias de las puntuaciones obtenidas en cada una de las subescalas por cada uno de los dos grupos de consumidores con las de su grupo control. También se compararon entre sí las medias obtenidas por el grupo de cocainómanos y el de heroinómanos. Se analizó la significación estadística de los resultados mediante el estudio de la diferencia de medias a través de la prueba T de Student para grupos independientes. Para realizar los cálculos se utilizó el programa SPSS.

\section{RESULTADOS Y DISCUSIÓN}

Una medida indirecta de la presencia de los trastornos de personalidad puede obtenerse por medio del
$\mathrm{TCl}$ a través de la categorización del Carácter como maduro o inmaduro (siendo en este caso muy alta la probabilidad de presentar un trastorno de la personalidad), por medio del valor de la suma de las puntuaciones obtenidas en las escalas S y C. Cloninger y cols. (1993) estimaron que puntuaciones inferiores a 57 indicarían un riesgo del $95 \%$ de tener un trastorno de personalidad. De esta manera inferimos que el $60 \%$ del grupo de estudio, tiene una alta probabilidad de presentar algún trastorno de personalidad específico. Este porcentaje coincide con las tasas descritas por otros autores. Así, Khantzian y Treece (1985) obtuvieron una tasa del $65 \%$ de trastornos de personalidad en una muestra de adictos a sustancias ilegales; Razzouk et al (2000) encuentran una proporción del $69 \%$ en usuarios de cocaína.

A continuación se describen los resultados encontrados en los diferentes grupos comparados:

\section{Dependientes a la heroína vs. población general}

El grupo de heroinómanos presenta diferencias tanto en las dimensiones de Temperamento como en las de Carácter frente a la población general. Tal y como se detalla en la Tabla 3, obtiene puntuaciones significativamente más bajas en las escalas de Dependencia de la Recompensa, Autodirección y Cooperación. También son significativas las divergencias encontradas en tres de las cuatro subescalas de Evitación del Riesgo ( $\uparrow H A 1$; 
$\downarrow H A 2 ; \uparrow H A 3)$ y en una de las correspondientes a Búsqueda de Novedades ( $\uparrow$ NS3).

Otros autores, que han estudiado con el TPQ (precursor de $\mathrm{TCl}$ ) poblaciones de adictos a opiáceos encuentran mayores diferencias en la dimensión Búsqueda de Novedades (Vukov et al 1995) (Gerra et al 2000) y resultados similares en las subescalas de Evitación del Riesgo y Dependencia de la Recompensa (Vukov op. cit.).

Los resultados por nosotros encontrados, referidos a las dimensiones del Temperamento, indicarían que el grupo de heroinómanos presenta características de personalidad heredadas diferenciales con relación a la población general. Así, la menor necesidad de recompensa apunta a que tienen menos sensibilidad ante los sentimientos de los demás y mayores dificultades para establecer contacto social ( $\downarrow R D 1)$; mayor tendencia al aislamiento y a la introversión y menor preocupación por la aceptación y la aprobación social ( $\downarrow R D 3)$. Se presentan como más autosuficientes e independientes ( $\downarrow$ RD4). En relación con la Evitación del Riesgo, se presentan más confiados y despreocupados ante el futuro que la población general ( $\uparrow \mathrm{HA} 1)$, muestran menor preocupación ante situaciones nuevas ( $\downarrow$ HA2) y mayor atracción por las situaciones arriesgadas o peligrosas $(\uparrow$ HA3). En la dimensión Búsqueda de Novedades, destaca su menor previsión y cautela, así como tendencia a dar menos valor al dinero, desprendiéndose fácilmente de él y a apurar las situaciones hasta el límite ( NNS3).

Con relación a las dimensiones del Carácter, la menor Autodirección, indica que presentan mayores sentimientos de inferioridad y menor autoestima que la población general. Tienden a culpabilizar a los demás de sus problemas ( S1), son más inmaduros ( $\$$ S2) y tienen menor $n^{\circ}$ de recursos ( $\left.\downarrow S 5\right)$. Los resultados obtenidos en la Cooperación, les sitúan respecto a la población general, con un menor nivel de tolerancia social $(\downarrow C 1)$, mayor desinterés por los demás $(\downarrow C 2)$ y mayor tendencia a manipular las situaciones en beneficio propio ( $\downarrow$ C5).

\section{Dependientes a la cocaína vs. población general}

El grupo de cocainómanos presenta diferencias en dos de las cuatro escalas de Temperamento. Así, presentan mayor Búsqueda de Novedades y menor De-

Tabla $\mathbf{n}^{\circ}$ 3. Comparación de las puntuaciones* TCl del Grupo de Heroinómanos y el Grupo Control

\begin{tabular}{|c|c|c|c|c|c|}
\hline \multirow{2}{*}{$\begin{array}{l}\text { Sub- } \\
\text { escalas }\end{array}$} & \multirow{2}{*}{ Escalas } & \multicolumn{2}{|c|}{ Media $\pm D T$} & \multirow[b]{2}{*}{$\mathbf{T}$} & \multirow[b]{2}{*}{$\mathbf{P}$} \\
\hline & & GrupoEstudio & Grupo Control & & \\
\hline \multirow[b]{3}{*}{ NS3 } & Escalas del Temperamento & & & & \\
\hline & Búsqueda de Novedades (NS) & & & & \\
\hline & $\begin{array}{l}\text { Reserva vs Extravagancia } \\
\text { Evitación del riesgo (HA) }\end{array}$ & $6,2 \pm 1,8$ & $5,1 \pm 1,9$ & 2,88 & 0,005 \\
\hline HA1 & Optimismo vs Preocupación & $5,6 \pm 2,6$ & $4,3 \pm 2,3$ & 2,65 & 0,009 \\
\hline HA2 & Confianza vs Miedo a la incertidumbre & $4,1 \pm 2,1$ & $5,1 \pm 1,5$ & $-2,94$ & 0,004 \\
\hline \multirow[t]{2}{*}{ HA3 } & Gregarismo vs Timidez & $5,0 \pm 2,0$ & $3,5 \pm 1,9$ & 3,74 & 0,000 \\
\hline & Dependencia de la Recompensa (RD) & $14,0 \pm 4,1$ & $17,9 \pm 3,6$ & $-4,94$ & 0,000 \\
\hline RD1 & Insensibilidad vs Sentimentalidad & $6,0 \pm 2,1$ & $7,5 \pm 1,5$ & $-4,01$ & 0,000 \\
\hline RD3 & Desapego vs Apego & $4,7 \pm 2,2$ & $6,2 \pm 1,9$ & $-3,49$ & 0,001 \\
\hline \multirow[t]{3}{*}{ RD4 } & Independencia vs Dependencia & $3,3 \pm 1,5$ & $4,1 \pm 1,4$ & -3 & 0,003 \\
\hline & Escalas del Carácter & & & & \\
\hline & Autodirección (S) & $26,6 \pm 8,1$ & $31,0 \pm 7,7$ & $-2,75$ & 0,007 \\
\hline S1 & Tendencia a culpabilizarse vs Responsabilidad & $5,1 \pm 2,1$ & $6,1 \pm 1,8$ & $-2,35$ & 0,021 \\
\hline S2 & Ausencia de metas vs Claridad de ideas & $5,4 \pm 1,9$ & $6,2 \pm 1,8$ & -2 & 0,048 \\
\hline \multirow[t]{2}{*}{ S5 } & Congruencia & $6,3 \pm 2,7$ & $8,3 \pm 2,4$ & $-3,72$ & 0,000 \\
\hline & Cooperación (C) & $27,8 \pm 7,7$ & $32,7 \pm 4,4$ & $-3,89$ & 0,000 \\
\hline $\mathrm{C} 1$ & Intolerancia social vs Aceptación social & $5,7 \pm 1,9$ & $6,8 \pm 1,5$ & $-3,02$ & 0,003 \\
\hline $\mathrm{C} 2$ & Desinterés social vs Empatía & $4,3 \pm 1,6$ & $5,1 \pm 1,2$ & $-2,60$ & 0,011 \\
\hline $\mathrm{C} 4$ & Venganza vs Compasión & $6,6 \pm 2,9$ & $8,2 \pm 1,7$ & $-3,15$ & 0,002 \\
\hline C5 & Jugador de ventaja vs de buen corazón & $5,7 \pm 1,7$ & $6,7 \pm 1,2$ & $-3,10$ & 0,002 \\
\hline
\end{tabular}


pendencia de la Recompensa. También presenta significación los resultados de una de las subescalas de evitación al daño $(\downarrow H A 2)$. Con relación a las escalas de Carácter, el grupo de estudio difiere del control en que presenta menor Autodirección y Cooperación. Las puntuaciones para cada una de las escalas y subescalas con significación estadística se detalla en la Tabla 4.

En la revisión bibliográfica realizada, no hemos encontrado estudios que investiguen con la misma prueba las dimensiones de personalidad de los cocainómanos. Sin embargo, el resultado por nosotros encontrado en la dimensión búsqueda de novedad, es coincidente con la que describen otros autores para el colectivo de adictos a opiáceos. (Vukov op.cit.) (Gerra op.cit.)

En el grupo de cocainómanos, encontramos también características de personalidad que les diferencian de la población general, incluso en mayor medida que en el grupo de heroinómanos. Sobre todo en lo que tiene que ver con los rasgos relacionados con el Temperamento.

Presentan una mayor Búsqueda de Novedades. Esto es indicativo de que, en relación con la población general, son más impulsivos que reflexivos a la hora de tomar decisiones ( $\uparrow$ S2), necesitan buscar mayor número de estímulos para no caer en el aburrimiento ( $\uparrow S 3)$, evitan las situaciones que les frustran y necesitan activamente refuerzos inmediatos para mantener las conductas, son capaces de saltarse las normas y se manejan bien en la ambigüedad ( $\uparrow$ S4). Las menores puntuaciones obtenidas en Dependencia de la Recompensa, corresponden a personas con un nivel de "practicidad" muy alto y que no se conmueven fácilmente $(\downarrow R D 1)$. Tienden al aislamiento y tienen dificultad para entablar relaciones íntimas ( $\downarrow R D 3)$. En relación a la escala Evitación del Riesgo, la diferencia significativa se circunscribe a la subescala confianza vs. Incertidumbre $(\downarrow H A 2)$, indicando un mayor nivel de atracción por el riesgo que los sujetos control.

Con relación a las dimensiones de Carácter, destacar que los resultados son similares a los del grupo de heroinómanos, por lo que los rasgos de personalidad diferenciales frente a la población general para ellos comentados, son también aplicables a este grupo. ( $\downarrow S ; \downarrow$

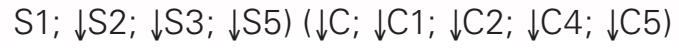

Tabla $n^{\circ}$ 4. Comparación de las puntuaciones* TCl del Grupo de Cocainómanos y el Grupo Control

\begin{tabular}{|c|c|c|c|c|c|}
\hline \multirow{2}{*}{$\begin{array}{l}\text { Sub- } \\
\text { escalas }\end{array}$} & \multirow{2}{*}{ Escalas } & \multicolumn{2}{|c|}{ Media $\pm D T$} & \multirow[b]{2}{*}{$\mathbf{T}$} & \multirow[b]{2}{*}{$\mathbf{P}$} \\
\hline & & Grupo Estudio & Grupo Control & & \\
\hline & Escalas del Temperamento & & & & \\
\hline & Búsqueda de Novedades (NS) & $22,3 \pm 5,5$ & $19,0 \pm 4,8$ & 3,74 & 0,000 \\
\hline NS2 & Reflexión vs Impulsividad & $5,1 \pm 2,3$ & $3,8 \pm 2,3$ & 3,11 & 0,002 \\
\hline NS3 & Reserva vs Extravagancia & $5,9 \pm 1,9$ & $4,8 \pm 1,8$ & 3,35 & 0,001 \\
\hline NS4 & $\begin{array}{l}\text { Reglamentación estricta vs Desorden } \\
\text { Evitación del riesgo (HA) }\end{array}$ & $5,2 \pm 1,9$ & $3,9 \pm 1,8$ & 3,96 & 0,000 \\
\hline \multirow[t]{2}{*}{ HA2 } & Confianza vs Miedo a la incertidumbre & $3,7 \pm 1,9$ & $4,9 \pm 1,6$ & $-3,65$ & 0,000 \\
\hline & Dependencia de la Recompensa (RD) & $13,7 \pm 3,7$ & $17,3 \pm 3,6$ & $-5,65$ & 0,000 \\
\hline RD1 & Insensibilidad vs Sentimentalidad & $6,2 \pm 2,2$ & $7,5 \pm 1,9$ & $-3,47$ & 0,001 \\
\hline \multirow[t]{3}{*}{ RD3 } & Desapego vs Apego & $4,0 \pm 2,1$ & $5,9 \pm 1,9$ & $-5,20$ & 0,000 \\
\hline & Escalas del Carácter & & & & \\
\hline & Autodirección (S) & $24,5 \pm 8,3$ & $31,3 \pm 6,4$ & $-5,37$ & 0,000 \\
\hline S1 & Tendencia a culpabilizarse vs Responsabilidad & $4,4 \pm 2,4$ & $6,1 \pm 1,6$ & $-4,79$ & 0,000 \\
\hline S2 & Ausencia de metas vs Claridad de ideas & $4,8 \pm 1,9$ & $6,4 \pm 1,5$ & $-5,19$ & 0,000 \\
\hline S3 & Apatía vs Recursos & $2,5 \pm 1,5$ & $3,3 \pm 1,3$ & $-3,40$ & 0,001 \\
\hline \multirow[t]{2}{*}{ S5 } & Congruencia & $6,2 \pm 2,6$ & $8,2 \pm 2,5$ & $-4,52$ & 0,000 \\
\hline & Cooperación (C) & $27,4 \pm 7,1$ & $32,7 \pm 5,3$ & $-4,83$ & 0,000 \\
\hline C1 & Intolerancia social vs Aceptación social & $5,7 \pm 1,9$ & $6,7 \pm 1,5$ & $-3,03$ & 0,003 \\
\hline C2 & Desinterés social vs Empatía & $4,1 \pm 1,4$ & $5,1 \pm 1,3$ & $-4,49$ & 0,000 \\
\hline C4 & Venganza vs Compasión & $6,6 \pm 2,9$ & $8,3 \pm 2,0$ & $-3,94$ & 0,000 \\
\hline C5 & Jugador de ventaja vs de buen corazón & $5,4 \pm 1,9$ & $6,8 \pm 1,3$ & $-4,76$ & 0,000 \\
\hline
\end{tabular}




\section{Dependientes a la cocaína vs. dependientes a la heroína}

Al igual que en otros estudios, encontramos que las similitudes entre las características de personalidad de los dependientes a opiáceos y a cocaína, son mayores que las diferencias. (Craig, 1988) (Craig y Olson, 1990) (Calsyn , 1990)

Tal y como se aprecia en la tabla 5, ambos grupos sólo difieren en dos de las subescalas del Temperamento: una perteneciente a la escala de Búsqueda de Novedades (NS4) y otra a la de Evitación del Riesgo (HA3). Así el grupo de cocainómanos presenta puntuaciones más altas en la subescala NS4 (Desorden) y puntuaciones más bajas en HA3 (Timidez) que el grupo de heroinómanos.

En cuanto a las características diferenciales entre ambos grupos, destacamos sobre la base de los resultados que los cocainómanos son más asertivos y con mejor nivel de competencia social que los heroinómanos ( $\downarrow$ HA3). Los heroinómanos presentan mayor nivel de improvisación y desenvoltura en situaciones de ambigüedad y que supongan el uso de mentiras ( $\downarrow$ NS4).

Tanto el grupo de adictos a la heroína como el de adictos a la cocaína, difieren significativamente de la población general con relación a las dimensiones de Carácter: autodirectividad y Cooperación. Las puntuaciones bajas en estas dimensiones correlacionan con las categorías de trastornos de personalidad.

\section{CONCLUSIONES}

- El 60 \% de la población drogodependiente estudiada tiene una alta probabilidad de presentar algún trastorno de personalidad tal y como los define el
DSM-IV, dadas las puntuaciones que obtiene en dos de las escalas de Carácter: autodirectividad y cooperación.

- En los dos grupos de drogodependientes estudiados, adictos a la cocaína y adictos a la heroína, se encuentran diferencias significativas con respecto a la población general tanto en las dimensiones de Temperamento (rasgos heredados) como en las de Carácter (rasgos añadidos).

- Las amplias diferencias encontradas entre el colectivo de drogodependientes y la población general, tanto en lo referido al substrato biológico de la personalidad (Temperamento) como al psicosocial (Carácter), corroboran la necesidad de mantener una postura integradora de los tratamientos biologicistas y psicoterapéuticos en los programas de rehabilitación.

- El pequeño $n^{\circ}$ de diferencias encontradas al comparar el grupo de heroinómanos y cocainómanos entre sí apuntan en la línea de los estudios que refutan la hipótesis de la elección de sustancia basándose en el substrato de personalidad.

- Haber tenido que excluir al colectivo de mujeres de la muestra estudiada por razones de significación estadística es una limitación del estudio. Sería interesante, recoger una muestra de mujeres suficientemente amplia para futuros estudios, que permitiera incorporar la perspectiva de género a la investigación del Temperamento y del Carácter del colectivo toxicómano. Quizá de ello se puedan establecer algunas pistas que permitan adaptar mejor los tratamientos a la población drogodependiente femenina y mejorar las tasas de retención.

- Teniendo en cuenta la comorbilidad de algunos diagnósticos en la población toxicómana, sería muy interesante realizar otros estudios con esta misma

\section{Tabla ${ }^{\circ}{ }^{5}$. Comparación de las puntuaciones* TCl del Grupo de Cocainómanos y el Grupo de Heroinómanos}

\begin{tabular}{|c|c|c|c|c|c|}
\hline \multirow{2}{*}{$\begin{array}{l}\text { Sub- } \\
\text { escalas }\end{array}$} & \multirow{2}{*}{ Escalas } & \multicolumn{2}{|c|}{ Media $\pm D T$} & \multirow[b]{2}{*}{$\mathbf{T}$} & \multirow[b]{2}{*}{$\mathbf{P}$} \\
\hline & & G. Cocainóm. & G. Heroinnóm. & & \\
\hline & Escalas del Temperamento & & & & \\
\hline NS4 & $\begin{array}{l}\text { Búsqueda de Novedades (NS) } \\
\text { Reglamentación estricta vs Desorden }\end{array}$ & $5,22 \pm 1,98$ & $4,41 \pm 2,01$ & 2,17 & 0,032 \\
\hline HA3 & $\begin{array}{l}\text { Evitación del riesgo (HA) } \\
\text { Gregarismo vs Timidez }\end{array}$ & $3,82 \pm 2,33$ & $5,02 \pm 2,00$ & $-2,89$ & 0,005 \\
\hline
\end{tabular}


prueba que establecieran correlaciones con los resultados obtenidos por los pacientes de otras categorías diagnósticas (Trastornos Afectivos, T. Psicóticos especialmente)

\section{REFERENCIAS}

Calsyn DA, Saxon AJ. (1990). Personality disorder subtypes among cocaine and opioid addicts using the Millon Clinical Multiaxial Inventory. Int. J. Addict. Vol. 25 (9), 1037-1049.

Cloninger CR. Svrakic DM, Przybeck TR. (1993) A psychobiological model of temperament and character. Archive of General Psichiatry Vol. 50, 975-990.

Craig, RJ. (2000): Prevalence of Personality Disorders among Cocaine and Heroin Addicts. Journal of substance abuse treatment. Vol.21 (2), 87-94.

Craig RJ., Olson Ref., (1990): MCMI comparisons of cocaine abusers and heroin addicts. Journal Clinical Psychology. Vol. 46 (2), 230-237.

Franques P., Auriacombe M., Tignol J. (2000): Adicción y personalidad. Encephale. Vol. 26 (1) 68-78.
Gutiérrez F, Torrens M, Boget T, Martín-Santos R, Sangorrin J, Pérez G, Salamero M. (2001). Psychometric properties of the Temperament and Character Inventory (TCl) questionnaire in Spanish psychiatric population. Acta Psychiatr Scand. Vol. 103 (2), 143-147.

Khantzian E.J., Treece C. (1985). DSM-III psychiatry diagnosis of narcotic addicts: Recent findings. Archives of General Psychiatry. Vol. 42, 1067-1071.

Mateos M, de la Gándara J. J.(2001): Temperamento, Carácter, Personalidad. Editorial SCM. Madrid, 2001.

Mateos M, Bayón C, de la Gándara J. J.(2001): TCI. Aplicación informática para el tratamiento de datos, corrección del test y emisión de informes. Editorial SCM. Madrid, 2001.

Razzouk D., Bordin IA., Jorge MR. (2000): Comorbidity and global functioning (DSM-III-R el Eje V) in a Brazilian sample of cocaine users. Journal of substance use and misuse. Vol.35 (9). 1307-1315.

Verhuel R. (2001). Comorbilidad de trastornos de la personalidad en individuos con trastorno por uso de sustancias. Eur Psychiatry (Ed Esp). Vol. 8, 520-529.

Vukov M., Baba-Milkic N., Lecic D, Mijalkovic S., Marinkovic J., (1995): Personality dimensions of opiate addicts. Acta Psychiatr Scand. Vol. 91 (2), 103-107. 